\title{
Comparison of the Radiologic, Morphometric, and Clinical Outcomes between Kinematically and Mechanically Aligned Total Knee Arthroplasty: A Propensity Matching Study
}

Tae Woo Kim, MD, PhD ${ }^{1}$ Jae Ik Lee, MD ${ }^{2}$ Han Gyeol Choi, MD ${ }^{2}$ Hyun Jin Yoo, MD ${ }^{2}$ Kyu Tae Kim, MD² Yong Seuk Lee, MD, $\mathrm{PhD}^{2}$

\footnotetext{
${ }^{1}$ Department of Orthopedic Surgery, Seoul National University College of Medicine, Seoul National University Boramae Medical Center, Seoul, The Republic of Korea

2 Department of Orthopedic Surgery, Seoul National University College of Medicine, Seoul National University Bundang Hospital, Seongnam, The Republic of Korea
}

\author{
Address for correspondence Yong Seuk Lee, MD, PhD, Department of \\ Orthopaedic Surgery, Seoul National University College of Medicine, \\ Bundang Hospital, 166 Gumi-ro, Bundang-gu, Seongnam-si, \\ Gyeonggi-do 463-707, The Republic of Korea \\ (e-mail: smcos1@hanmail.net).
}

J Knee Surg 2022;35:1453-1461.

\begin{abstract}
Keywords

- total knee arthroplasty

- kinematic alignment

- mechanical alignment

- coronal alignment

- rotational alignment

- morphometry

The purpose of this study was to compare radiologic, morphometric, and clinical outcomes between kinematically aligned (KA) and mechanically aligned (MA) total knee arthroplasty (TKA) in Korean patients. Overall, 168 patients who underwent primary TKA were retrospectively reviewed, and propensity matching (age, sex, and body mass index) was performed as 1:3 ration (KA TKAs [ $n=42]$ : MA TKAs $[n=126]$ ). Joint-line orientation angle (JLOA), coronal and axial alignments of implants, hip-knee-ankle (HKA) angle, and patellar tilt angle were assessed using full-length standing radiograph, axial computed tomography (CT) scan, and plain radiographs. Morphometric assessment was performed by analyzing the intraoperative measurement of the femoral cut surface and femoral components fitting in five zones. Clinical outcomes more than 2 years of follow-up were evaluated with the Knee Society (KS) knee and functional scores, Western Ontario and McMaster Universities Osteoarthritis Index (WOMAC) scores, and the Short-Form Health Survey (SF-36). In radiologic results, JLOA was more parallel to the floor in KA TKAs (KA: medial tilt $0.9 \pm 1.5$ degrees; MA: lateral tilt $1.7 \pm 1.5$ degrees, $p<0.05$ ), and patellar tilt angle was closer to preoperative status after KA TKA (KA: $2.0 \pm 1.6$ degrees; $\mathrm{MA} ; 0.3 \pm 1.2$ degrees, $p<0.05)$. HKA angle and rotational mismatch were similar between two groups. In morphometric analysis, entire overhang of anterior femoral cutting surface was reduced in KA TKA compared with MA TKA (KA: $11.7 \pm 6.2 \mathrm{~mm}$; MA: $14.4 \pm 5.9 \mathrm{~mm}$, $p<0.05)$. However, both of MA and KATKAs showed underhang in mediolateral dimension without difference. There were no significant differences in clinical scores between two groups. KA TKA showed more parallel JLOA to floor, closer patellar tilt to preoperative status, and better anterior flange fitting that can reproduce more natural knee kinematics compared with MA TKA. Although clinical outcomes assessed by conventional evaluating tools were similar between two groups, further evaluation focusing on the patellofemoral symptoms or unawareness of TKA is necessary to clarify the clinical benefit of KA TKA.
\end{abstract}

received

July 29, 2020

accepted after revision

January 12, 2021

published online

March 3, 2021 (c) 2021. Thieme. All rights reserved.

Thieme Medical Publishers, Inc., 333 Seventh Avenue, 18th Floor, New York, NY 10001, USA
DOI https://doi.org/

10.1055/s-0041-1725006. ISSN 1538-8506. 
Mechanical alignment is regarded as the current standard for the restoration of coronal alignment in total knee arthroplasty (TKA) and transepicondylar axis is generally accepted as a reliable landmark for the femoral component rotation. ${ }^{1,2}$ Regarding tibial component rotation, it is determined by several landmarks or axes including tibial anterior curved cortex and tibial tuberosity because there is lacking in consensus. ${ }^{3}$ The concept of anatomic restoration has gained interest in the form of kinematically aligned (KA) TKA. KA TKA corrects the arthritic deformity to correspond to the constitutional alignment of the patient, restoring the natural articular surface, and the laxities of the knee. ${ }^{4,5}$ Comparative studies between KA and mechanically aligned (MA) TKAs have shown that clinical outcomes of KA TKA were similar or better than those of MA TKA at early- to mid-term follow-up. ${ }^{6}$ Limb and knee alignments were similar to those of MA TKA, and component alignment of KA TKA showed slightly more varus in the tibial component and slightly more valgus in the femoral component. ${ }^{7}$ However, studies on the difference in axial rotation, and patellofemoral relation between MA and KA TKAs are limited.

Matching the patient's anatomy with TKA component was the goal of arthroplasty to achieve optimal results. ${ }^{8,9}$ The anthropometric measurement of distal femur cutting surface may be changed according to the alignment profile characteristics of KA and MA TKA. Especially, anterior cutting surface could be definitely different because they have different concepts in rotational alignment. While MA TKA encourage external rotation of the femoral component based on the transepicondylar axis, KA TKA insists neutral rotation of the femoral component. However, anthropometrical comparison between MA and KA TKAs are also rarely investigated yet. Therefore, it is necessary to evaluate whole alignment changes including coronal, sagittal, and axial planes on both MA and KA TKAs, and it is also sure to know how they produce different morphometries on the cutting surface.
The purpose of this study was to compare radiologic, morphometric, and clinical results between KA and MA TKAs in Asian patients. The hypotheses of this study were alignment profiles and morphometry of the femoral cutting surface between KA and MA TKAs would be different and this will produce different radiologic, morphometric, and clinical results.

\section{Materials and Methods}

\section{Patients}

We retrospectively reviewed 396 patients who underwent primary TKA at our institution between March 2017 and February 2018. The inclusion criteria were limited to patients with varus osteoarthritis (OA) of the knee with mild flexion contracture and varus deformity. The exclusion criteria were as follows: (1) OA with valgus deformity, (2) severe varus deformity (hip-knee-ankle [HKA] angle $>15$ degrees) or flexion contracture ( $>20$ degrees), (3) bone defects more than $5 \mathrm{~mm}$, (4) conversion TKA following osteotomy or unicompartmental knee arthroplasty, and (5) revisional TKAs. Forteen knees were excluded based on the exclusion criteria. Then, considering the KA and MA TKAs' incidence in our clinic, 1:3 propensity matching was performed based on the age, sex, and body mass index (BMI). Finally, a total of 168 patients (42 KA TKAs and 126 MA TKAs) were enrolled in this study, and evaluated using radiologic, morphometric, and clinical results with a minimum 2-year follow-up (-Fig. 1). This study was approved by the institutional review board of our institution.

\section{Radiological Evaluation}

Radiological evaluations were performed preoperatively and postoperative 1 year. Coronal alignment parameters including HKA angle, varus/valgus angle of the femoral and tibial components, and joint-line orientation angle (JLOA) in relation

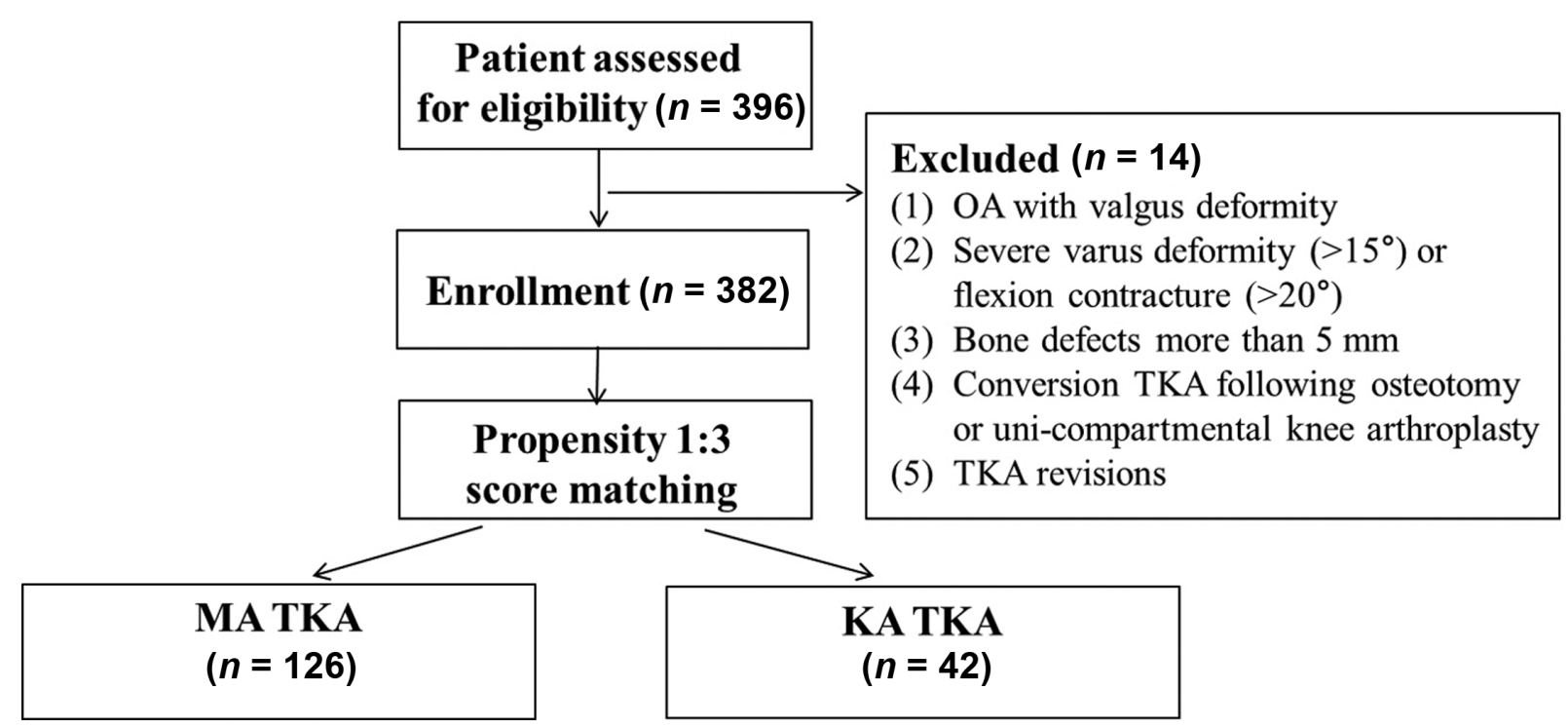

Fig. 1 Patient diagram of this study. OA, osteoarthritis; KA, kinematically aligned; MA, mechanically aligned; TKA, total knee arthroplasty. 

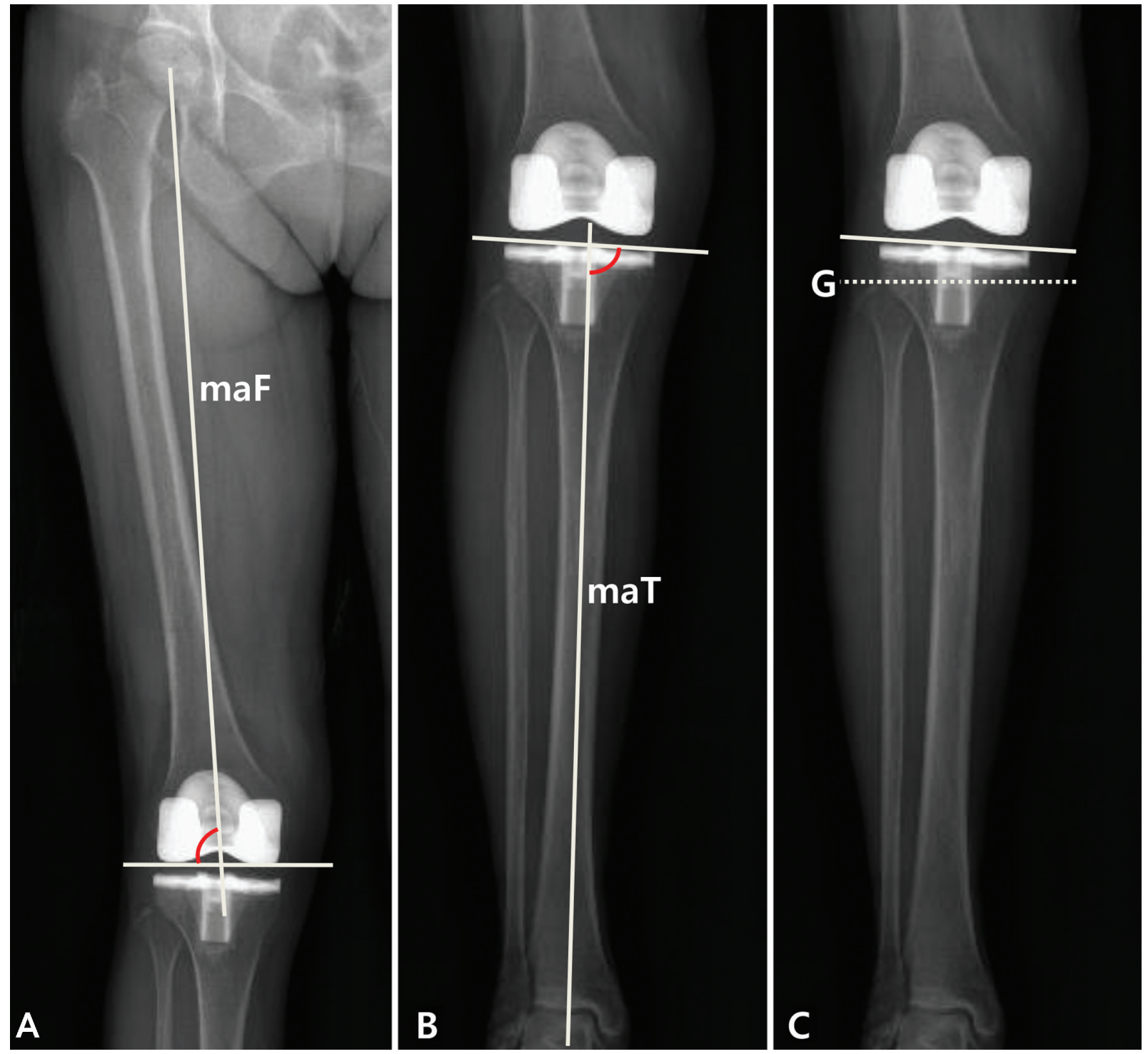

Fig. 2 Measurement of tibial component rotation: $(A-C)$ tibial component rotation was defined as the angle between the line that connect geometric center of tibial plate and medial margin of tibial tuberosity (tAP) and anterior-posterior axis of tibial component (tcAP). Internal rotation (-), external rotation (+). Dotted line in (C) represents orientation of ground (G). GC, geometric center; maF, mechanical axis of femur; maT, mechanical axis of tibia; mTT, medial margin of tibial tuberosity; tAP, AP axis of tibia; tcAP, AP axis of tibial component.

to the floor were analyzed with standing long-leg anteriorposterior (AP) radiographs obtained at the patellar facing forward position $^{10}$ (-Fig. 2). To evaluate patellofemoral relationship, patellar tilt angle was measured on axial plain radiograph. ${ }^{11}$

Rotational alignment of the femoral and tibial components and mismatch angle between the long axes of the two components were measured on postoperative axial computed tomography (CT) scans. Femoral component rotation was defined as the angle between the transverse axis of femoral component and the surgical transepicondylar axis. ${ }^{12}$ The rotation of the tibial component was defined as the angle between the line that connects the geometric center of the tibial plate and the medial margin of the tibial tuberosity and AP axis (- Fig. 3). ${ }^{13}$ Rotational mismatch was measured as the angle between AP axes of the femoral and tibial components. Tibial component rotation and rotational mismatch was measured by overlapping of the two axial transparent CT images (-Fig. 4). To evaluate intra- and interobserver reliabilities of the radiological assessment, two orthopaedic surgeons measured all radiological parameters twice with intervals of 6 weeks.

\section{Morphometric Analysis}

Morphometric assessment was performed by analyzing the intraoperative measurement of the femoral cut surface, and femoral components fitting in five zones. ${ }^{14}$ Measurements for zones A and B were defined as length of anterior medial (LAM) and length of anterior lateral (LAL) condyles. The measured distance was the perpendicular distance from the anterior cut surface to the most proximal point of the resected surfaces. Zones A and B were related with the morphometry of the anterior cut surface. Anterior flange width (AFW, zone C) was measured to evaluate anterior 


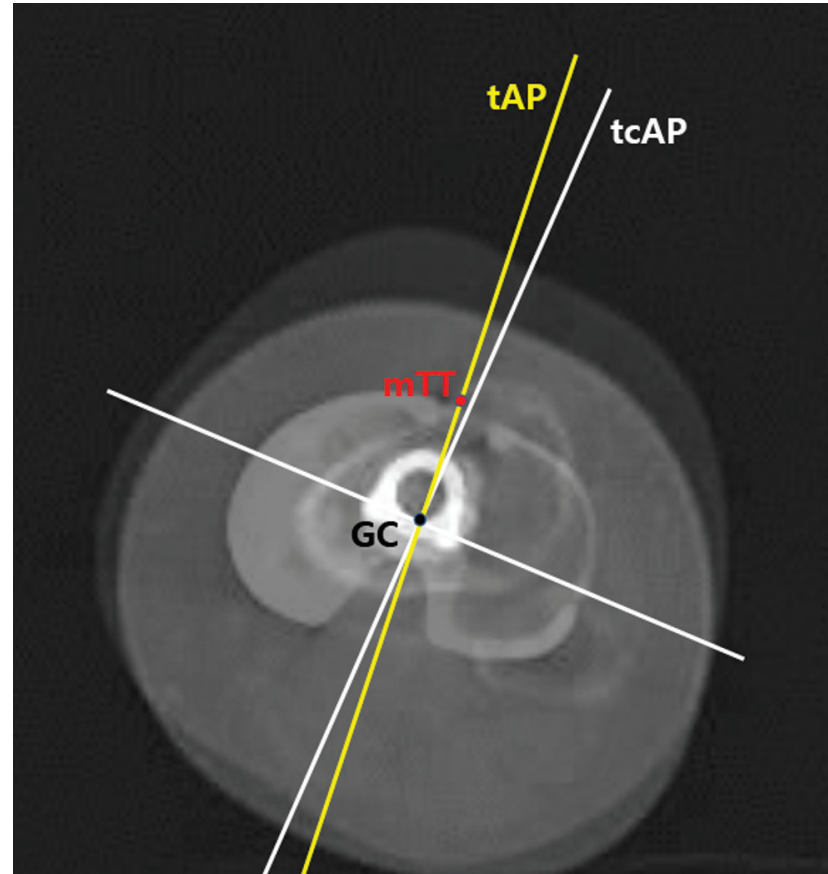

Fig. 3 Measurement of rotational mismatch between femoral and tibial components: rotational mismatch angle was measured as the angle between anterior-posterior axes of femoral (fcAP) and tibial components (tcAP). mTT, medial margin of tibial tuberosity.

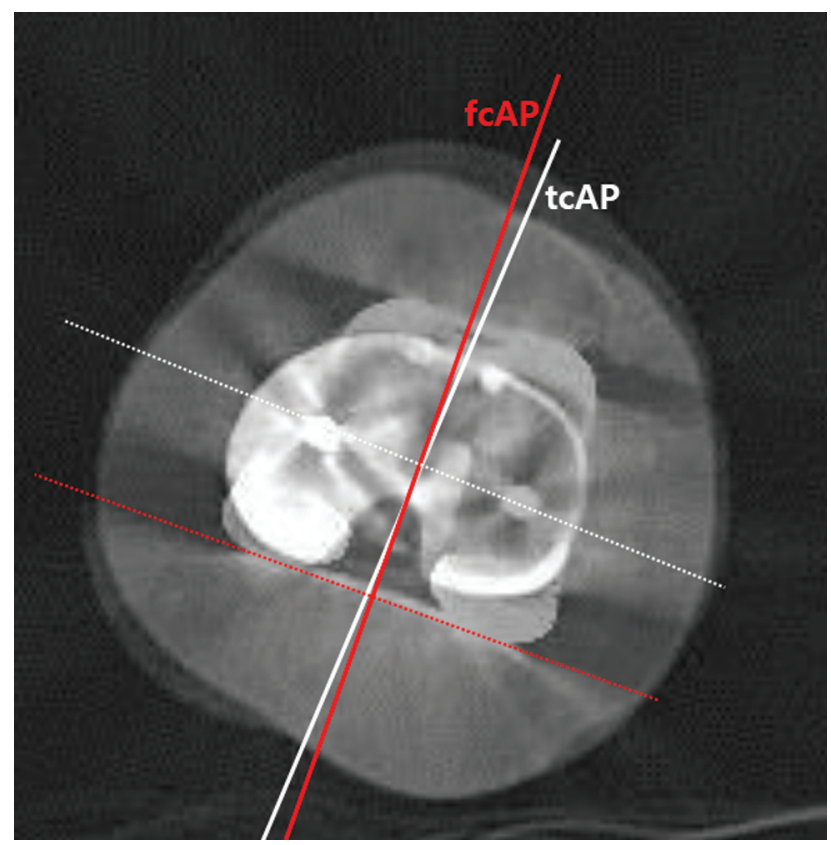

Fig. 4 Measurement of distal femoral cutting surfaces of right knee. fcAP, anterior-posterior axis of femoral component; tcAP, anteriorposterior axis of tibial component.

flange mediolateral dimension. Middle mediolateral width (MML, zone D) and posterior medial-lateral width (PML, zone E) were related to the distal cutting surface (-Fig. 5). The dimensions from each zone were compared with the corresponding dimension of the prosthesis supplied by the manufacturer. The difference between the prosthesis dimension and the actual femoral dimension was deemed positive when there is oversizing, and negative when undersized.

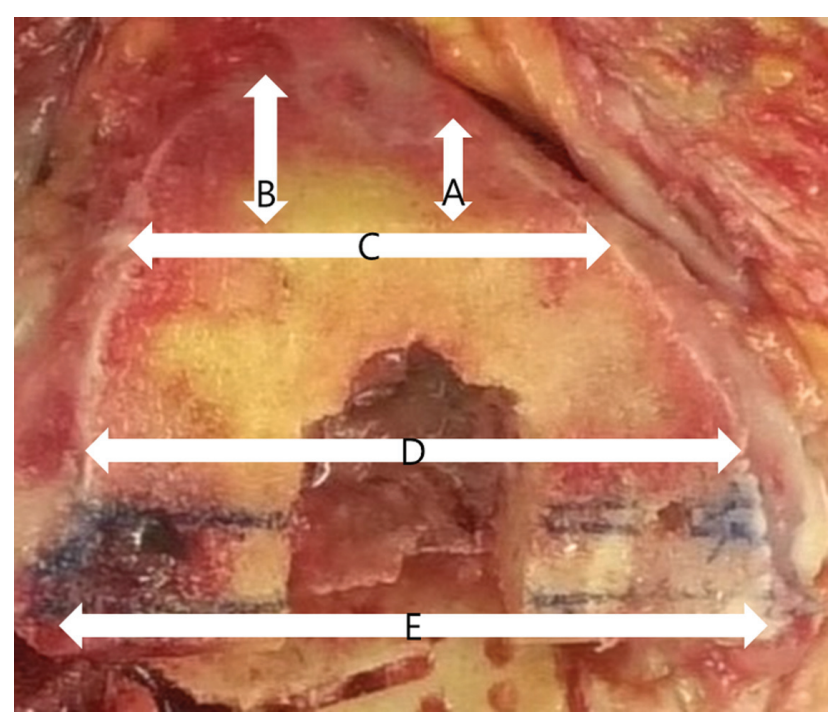

Fig. 5 (A) zone 1: length of anteromedial condyle (LAM); (B) zone 2: length of anterolateral condyle (LAL); (C) zone 3 : anterior flange width (AFW); (D) zone 4: middle medial-lateral width (MML); (E) zone 5: posterior medial-lateral width (PML).

Sum of cut surface-prosthesis difference in zones A and B was also measured to evaluated entire fitting of anterior flange between two groups. All measurements were performed by a single senior surgeon with the consensus of fellows during the operation.

\section{Clinical Evaluation}

Preoperatively and minimum 2 years postoperatively, clinical results were rated using the Knee Society (KS) knee and functional scores, Western Ontario and McMaster Universities Osteoarthritis Index (WOMAC) scores, and the ShortForm Health Survey (SF-36). Range of motion (ROM; flexion contracture and maximum active flexion angle) was evaluated preoperatively and postoperatively at 6 months, 1 year, and 1-year interval thereafter. All clinical evaluations were performed by physical assistant who was blinded to the operation.

\section{Surgical Technique}

All TKAs were performed by a single surgeon who had more than 10 years' experience of TKA. In all patients, Persona knee implants (Zimmer Biomet, Warsaw, IN) were used with the cement fixation technique. The patella was resurfaced selectively depending on the arthritic status and thickness of the patella.

For KA TKA, there was minimal release of the deep medial collateral ligament (MCL). All procedures regarding bone resection was performed based on the Howell's KA TKA technique using conventional instrument. ${ }^{15}$ To make equal 9-mm cuts of the medial and lateral distal femur including the cartilage thickness, cartilage wear on the medial distal femoral condyle was judged and full-thickness wear was regarded as $2 \mathrm{~mm}$ and partial thickness as $1 \mathrm{~mm}$ with a saw thickness of $1 \mathrm{~mm}$. After additional 1 - or $2-\mathrm{mm}$ sim was attached to the medial surface of distal cutting guide, distal femur was resected locating the cutting guide parallel to the 
Table 1 Thickness of tibial and femoral bone cuts during kinematically aligned total knee arthroplasty

\begin{tabular}{|l|l|l|}
\hline & & $\begin{array}{l}\text { Thickness of } \\
\text { bone cut }(\mathrm{mm})\end{array}$ \\
\hline Tibia & Medial & $5.4 \pm 1.8$ \\
\hline & Lateral & $7.9 \pm 1.7$ \\
\hline Distal femur & Medial & $6.8 \pm 0.8$ \\
\hline & Lateral & $8.0 \pm 0.5$ \\
\hline $\begin{array}{l}\text { Posterior } \\
\text { femur }\end{array}$ & Medial & $7.9 \pm 0.6$ \\
\hline & Lateral & $7.8 \pm 0.4$ \\
\hline
\end{tabular}

joint line. Adjustment of intramedullary guide angle based on the preoperative measurements on long-leg radiograph and wide proximal reaming enabled distal cutting guide to contact both sides of distal femur simultaneously. To verify the cutting accuracy, thickness of resected bone was measured using digital caliper (- Table 1). The rotational axis of tibia was determined as the AP axis of the lateral tibial plateau and tibial cutting was performed to make equal 9mm cutting of medial and lateral tibial plateau considering cartilage wear and 7 degrees of posterior slope. The femoral component rotation and size were determined using posterior referencing guide with 0 degrees of external rotation and all cases showed relatively intact posterior cartilage thickness. Thus, distal and AP femoral and tibial cuttings were performed to reproduce native joint orientation.

For the MA TKA, distal femur was resected perpendicular to mechanical axis and femoral component was rotated 3degree externally in all cases. Tibial bone was also resected perpendicular to mechanical axis, aiming within 5 degrees of posterior slope. Femoral AP cutting was performed with anterior referencing system. Tibial rotation was determined by first adjusting to the posterolateral corner and secondarily adjusting to the anterior cortical rim. Compared with the KA TKA, extension and flexion gaps were all targeted to the rectangular gap. Ligament balancing was performed by selective piecrusting in all cases.

\section{Statistical Analysis}

All parameters were presented as mean with standard deviation (SD). Categorical variables were analyzed with Pearson's Chi-squared test or Fisher's exact test and continuous variables were analyzed with Student's $t$-test. Potential confounding and selection biases were accounted for by developing a propensity score because patients were not randomly assigned. The propensity for surgical technique was determined using multivariable logistic regression analysis. After calculating the propensity scores for the three independent variables of age, sex, and BMI, a 1:3 match was performed. The statistical significance was set at $p<0.05$. Intraclass correlation coefficients were used to evaluate intra- and interobserver reliability. All statistical analyses were performed with SPSS (version 22.0, Chicago, IL).
Table 2 Comparison of preoperative demographic data between two groups using propensity matching

\begin{tabular}{|l|l|l|l|}
\hline & KA $(\boldsymbol{n}=\mathbf{4 2})$ & MA $(\boldsymbol{n}=126)$ & $p$-Value \\
\hline Mean age $(\mathrm{y})$ & $70.30 \pm 5.06$ & $70.38 \pm 5.76$ & 0.937 \\
\hline Sex (M:F) & $2: 40$ & $9: 117$ & 0.592 \\
\hline $\begin{array}{l}\text { Laterality } \\
\text { (right/left) }\end{array}$ & $20 / 22$ & $59 / 67$ & 0.414 \\
\hline $\begin{array}{l}\text { Body mass } \\
\text { index }\left(\mathrm{kg} / \mathrm{m}^{2}\right)\end{array}$ & $25.11 \pm 3.31$ & $25.34 \pm 3.10$ & 0.481 \\
\hline HKA angle & Varus $7.7 \pm 4.5$ & Varus $8.4 \pm 5.5$ & 0.375 \\
\hline $\begin{array}{l}\text { ROM } \\
\text { (degrees) }\end{array}$ & $118.5 \pm 9.5$ & $116.7 \pm 12.4$ & 0.340 \\
\hline KS score knee & $26.6 \pm 10.4$ & $23.6 \pm 9.3$ & 0.165 \\
\hline function & $38.6 \pm 10.1$ & $36.1 \pm 6.3$ & 0.156 \\
\hline WOMAC & $49.0 \pm 8.8$ & $50.8 \pm 8.5$ & 0.354 \\
\hline SF-36 PCS & $28.3 \pm 6.7$ & $25.3 \pm 9.2$ & 0.118 \\
\hline MCS & $33.9 \pm 10.3$ & $30.7 \pm 7.7$ & 0.103 \\
\hline
\end{tabular}

Abbreviations: F, female; HKA, hip-knee-ankle; KA, kinematically aligned; KS, Knee Society; M, male; MA, mechanically aligned; MCS, mental component summary; PCS, physical component summary; ROM, range of motion; SF, Short Form; WOMAC, Western Ontario and McMaster Universities.

Note: Values are presented as mean \pm standard deviation. The statistical significance was set at $p<0.05$.

\section{Results}

The inter- $(\mathrm{kappa}=0.831)$ and intraobserver $(\mathrm{kappa}=0.859)$ reliabilities were good. There were no significant differences in terms of demographics and preoperative clinical scores (-Table 2). Preoperative varus deformity and range of motion were not different between two groups by propensity matching. The mean follow-up periods were $28 \pm 3.5$ months at KA TKA group and $28 \pm 4.2$ months at MA TKA group.

\section{Radiological Results}

In coronal plane, femoral component of KA TKAs were implanted with more valgus position compared with MA TKAs (KA: valgus $2.5 \pm 2.0$ degrees; MA: varus $0.8 \pm 1.8$ degrees, $p<0.05)$. On the contrary, tibial component of KA TKAs were implanted with more varus position compared with MA TKAs (KA: varus $2.5 \pm 1.5$ degrees; MA: varus $0.1 \pm 1.6$ degrees, $p<0.05$ ). However, there was no difference between two groups in the HKA angle that presented the entire coronal limb alignment (KA: varus $0.5 \pm 2.4$ degrees; MA: varus $1.3 \pm 2.1$ degrees, $p=0.163$ ). JLOA was more parallel to the floor in KA TKAs compared with MA TKAs (KA: medial tilt $0.9 \pm 1.5$ degrees; MA: lateral tilt $1.7 \pm 1.5$ degrees, $p<0.05$;

-Fig. 6).

In rotational alignment, both of the femoral and tibial components were rotated more internally in KA TKA than in MA TKA (femur, KA: internal rotation $[\mathrm{IR}]=1.1 \pm 1.4$ degrees, $M A$; external rotation [ER] $=0.5 \pm 1.7$ degrees, $p<0.05 /$ tibia, KA: $3.85 \pm 3.2$ degrees, MA: $5.32 \pm 3.4$ degrees, $p<0.05$ ). However, there was no significant difference in rotational mismatch angle between the two components (KA: $1.1 \pm 1.4$ degrees, MA: $0.9 \pm 1.2$ degrees, $p=0.35$; - Table 3 ). 


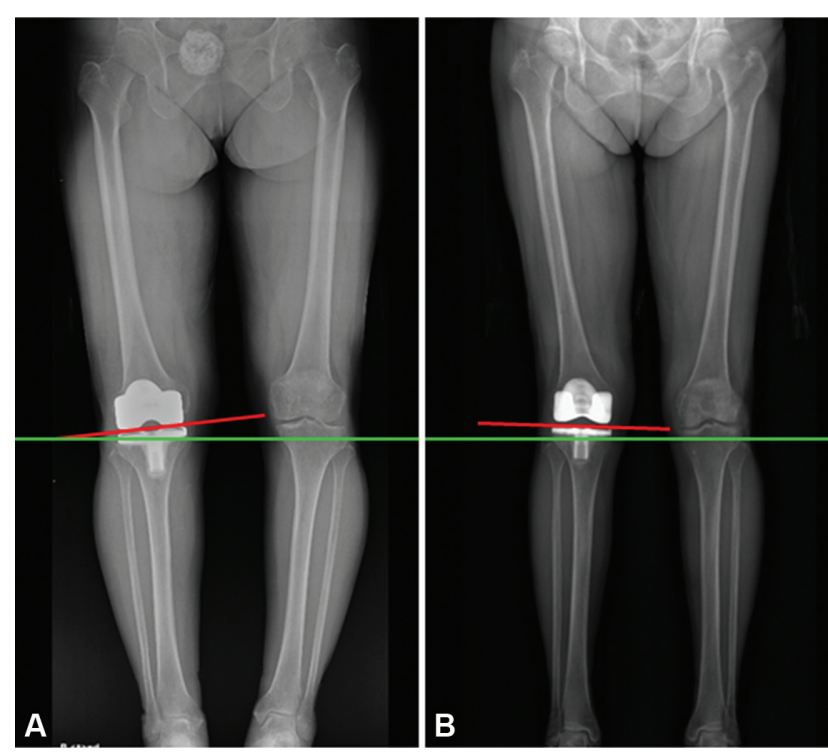

Fig. 6 Comparison of Joint-line orientation angle (JLOA) between mechanical aligned (MA) and kinematic aligned (KA) total knee arthroplasty (TKA). (A) Lateral tilt (negative value) JLOA in MA TKA; (B) Medial tilt (positive value) JLOA in KA TKA.

Patellar-resurfacing ratio were comparable between KA and MA groups (KA: 40.5\%, MA: 42.9\%). On axial plain radiograph, the patella was tilted more laterally in KATKA than in MATKA. However, patellar tilt angle was more close to preoperative status after KA TKA than after MA TKA (KA: $2.0 \pm 1.6$ degrees MA: $0.3 \pm 1.2$ degrees - Fig. 7).

\section{Morphometric Results}

The average length of distal femoral cutting surface increased in transitioning from zone A to zone E. Our results show that the maximal length of zone $B(L A L)$ was longer than the length of zone $A$ (LAM) in both of MA and KA groups. However, KA TKA showed longer zone A dimension (KA: $14.8 \pm 4.1 \mathrm{~mm}$; MA: $10.1 \pm 4.1 \mathrm{~mm}, p<0.05)$ and shorter zone $B$ dimension (KA: $21.9 \pm 5.1 \mathrm{~mm}$; MA: $23.4 \pm 5.0 \mathrm{~mm}, p<0.05$ ) compared with MA TKA. There were no statistically significant differences between MA and KATKA groups in the zones C, D, and E. When analyzing mean difference between implant and distal femoral cutting surface of the 168 knees, zones A and B showed overhang both of MA and KA groups. However, KATKA showed shorter mean difference of zone $A$ and implant (KA: $3.1 \pm 4.3 \mathrm{~mm}$; MA: $7.2 \pm 4.3 \mathrm{~mm}, p<0.05)$ and longer mean difference of zone $B$ and implant (KA: $8.6 \pm 4.1 \mathrm{~mm}$; MA: $7.2 \pm 4.0 \mathrm{~mm}, p<0.05$ ) compared with MA TKA. Considering entire anterior cut surface overhang, KA showed better anterior flange fitting than MA TKA (KA: $11.7 \pm 6.2 \mathrm{~mm}$; MA: $14.4 \pm 5.9 \mathrm{~mm}, p<0.05)$.

Zones C, D, and E showed underhang both of MA and KA groups. There were no statistically significant differences between MA and KA TKA groups in the zones C, D, and E (-Table 4).

\section{Clinical Results}

At the final follow-up, there was no significant difference in $\mathrm{KS}$ knee score and KS function score between KA and MA TKA (KS
Table 3 Comparison of radiologic analysis between two groups

\begin{tabular}{|c|l|l|l|}
\hline Alignment profiles & $\begin{array}{l}\text { KA } \\
(\boldsymbol{n}=\mathbf{4 2})\end{array}$ & $\begin{array}{l}\text { MA } \\
(\boldsymbol{n}=\mathbf{1 2 6})\end{array}$ & $p$-Value \\
\hline Coronal alignment & & & \\
\hline FC Alignment (degree) & $2.5 \pm 2.0$ & $-0.8 \pm 1.8$ & $<0.05$ \\
\hline TC Alignment (degree) & $2.5 \pm 1.5$ & $0.1 \pm 1.6$ & $<0.05$ \\
\hline JLOA (degree) & $-0.9 \pm 1.5$ & $1.7 \pm 1.5$ & $<0.05$ \\
\hline HKA angle (degree) & $0.5 \pm 2.4$ & $1.3 \pm 2.1$ & 0.163 \\
\hline Rotational alignment & & & \\
\hline FCR (s TEA; degree) & $1.1 \pm 1.4$ & $0.5 \pm 1.7$ & $<0.05$ \\
\hline TCR (degree) & $3.85 \pm 3.2$ & $5.32 \pm 3.4$ & $<0.05$ \\
\hline Mismatch (degree) & $1.1 \pm 1.4$ & $0.9 \pm 1.2$ & 0.355 \\
\hline $\begin{array}{l}\text { Patellar tilt angle } \\
\text { (degree) }\end{array}$ & & & \\
\hline Preoperative & $1.5 \pm 1.5$ & $1.3 \pm 1.7$ & 0.254 \\
\hline Postoperative & $2.0 \pm 1.6$ & $0.3 \pm 1.2$ & $<0.05$ \\
\hline $\begin{array}{l}\text { SPre-op-post op } \\
\text { (degree) }\end{array}$ & $0.5 \pm 1.7$ & $1.1 \pm 2.0$ & $<0.05$ \\
\hline
\end{tabular}

Abbreviations: FC, femoral component ; FCR, femoral component rotation ; HKA, hip-knee-ankle; IR,; JLOA, joint-line orientation angle; KA, kinematically aligned; MA, mechanically aligned; Pre-op, preoperative; post-op, postoperative; TC, tibial component ; TCR, tibial component rotation; TEA, surgical transepicondylar axis.

Notes: Values are presented as mean \pm standard deviation. The statistical significance was set at $p<0.05$.

FC alignment (degree) was defines as 90 - lateral distal femoral angle (degree). Positive and negative values of FC alignment means valgus and varus, respectively. TC alignment (degree) was defines as $90-$ medial proximal tibial angle (degree). Positive and negative values of TC alignment means varus and valus, respectively. Positive and negative values of JLOA means lateral and medial tilt, respectively. Positive and negative values of HKA angle means varus and valgus, respectively. Positive and negative values of FCR means external rotation and internal rotation, respectively.

knee, KA TKA: $82.1 \pm 6.4$, MA TKA: $80.2 \pm 6.7, p=0.529 /$ KS function, KA TKA: $85.0 \pm 7.5$, MA TKA: $82.7 \pm 6.4, p=0.471$ ). The WOMAC score and SF-36 score were also similar between two groups (WOMAC, KA TKA: $20 \pm 7.9$, MA TKA: $16 \pm 7.6$, $p=0.428 /$ SF-36, KA TKA: $83.1 \pm 6.3$, MA TKA: $83.5 \pm 8.2$ $p=0.921)$. For ROM, KA TKA showed increased flexion angle only at postoperative 3 months compared with MA TKA (KA: $134.2 \pm 10.3$ degrees; MA: $129.1 \pm 10.5$ degrees, $p=0.038)$. However, there was no difference in ROM between the two groups at postoperative 1 year. In all groups, there were no complications including implant loosening or infection and problematic instability that required revision surgery during the follow-up (-Table 5).

\section{Discussion}

The principal finding of this study was that KA TKA showed (1) more parallel joint line to the floor with femoral component valgus and tibial component varus, (2) closer patellar tilt to the preoperative status, and (3) more proper fitting of the anterior flange, compared with MA TKA. 

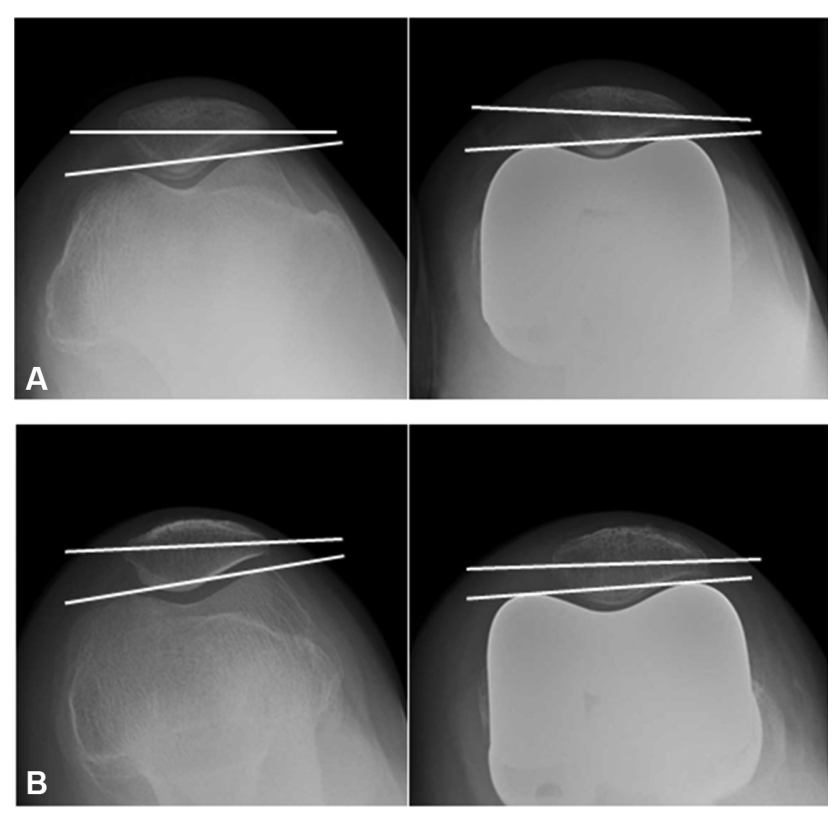

Fig. 7 Comparison of patellar tilt angle between mechanically aligned (MA) and kinematically aligned (KA) total knee arthroplasty (TKA). (A) Decreased patellar tilt in MA TKA compared with preoperative status; (B) Similar patellar tilt in KA TKA compared with preoperative status.

Table 4 Comparison of intraoperative morphometric analysis between two groups

\begin{tabular}{|c|c|c|c|}
\hline $\begin{array}{l}\text { Anthropometric } \\
\text { measurement }\end{array}$ & $\begin{array}{l}\mathrm{KA} \\
(n=42)\end{array}$ & $\begin{array}{l}\mathrm{MA} \\
(n=126)\end{array}$ & $p$-Value \\
\hline \multicolumn{4}{|c|}{$\begin{array}{l}\text { Distal femur } \\
\text { cutting surface }(\mathrm{mm})\end{array}$} \\
\hline Zone A & $14.8 \pm 4.1$ & $10.1 \pm 4.1$ & $<0.05$ \\
\hline Zone B & $21.9 \pm 5.1$ & $23.4 \pm 5.0$ & $<0.05$ \\
\hline Zone C & $48.2 \pm 4.2$ & $47.8 \pm 3.9$ & 0.510 \\
\hline Zone D & $60.1 \pm 2.2$ & $59.5 \pm 3.4$ & 0.246 \\
\hline Zone E & $68.8 \pm 2.9$ & $68.3 \pm 3.3$ & 0.703 \\
\hline \multicolumn{4}{|c|}{$\begin{array}{l}\text { Mean difference between implant and distal } \\
\text { femoral cutting surface }(\mathrm{mm})\end{array}$} \\
\hline Zone A & $3.1 \pm 4.3$ & $7.2 \pm 4.3$ & $<0.05$ \\
\hline Zone B & $8.6 \pm 4.1$ & $7.2 \pm 4.0$ & $<0.05$ \\
\hline Zone $\mathrm{A}+\mathrm{B}$ & $11.7 \pm 6.2$ & $14.4 \pm 5.9$ & $<0.05$ \\
\hline Zone C & $-3.2 \pm 2.3$ & $-3.0 \pm 3.6$ & 0.671 \\
\hline Zone D & $-4.3 \pm 3.7$ & $-3.8 \pm 3.1$ & 0.719 \\
\hline Zone E & $-5.2 \pm 3.9$ & $-4.9 \pm 3.1$ & 0.485 \\
\hline
\end{tabular}

Abbreviations: KA, kinematically aligned; MA, mechanically aligned. Note: Values are presented as mean \pm standard deviation. The statistical significance was set at $p<0.05$.

However, regardless of these differences, there was no significant difference in clinical outcomes between the two groups. Therefore, our hypotheses were partially adopted and partially declined.

Similar to recent meta-analyses that compared KA and MA TKAs, our study showed more valgus femoral component and more varus tibial component with comparable HKA angle in
Table 5 Comparison of clinical results of 2-year follow-up between two groups

\begin{tabular}{|l|l|l|l|}
\hline & $\begin{array}{l}\text { KA } \\
(\boldsymbol{n}=\mathbf{4 2})\end{array}$ & $\begin{array}{l}\text { MA } \\
(\boldsymbol{n}=\mathbf{1 2 6})\end{array}$ & $p$-Value \\
\hline KS score knee & $82.1 \pm 6.4$ & $80.2 \pm 6.7$ & 0.529 \\
\hline Function & $85 \pm 7.5$ & $82.7 \pm 6.4$ & 0.471 \\
\hline WOMAC scores & & & \\
\hline Total & $20 \pm 7.9$ & $16 \pm 7.6$ & 0.428 \\
\hline Pain & $2.8 \pm 2.3$ & $2 \pm 1.3$ & 0.424 \\
\hline Stiffness & $0.8 \pm 0.9$ & $0.5 \pm 0.8$ & 0.370 \\
\hline Function & $16.2 \pm 5$ & $15.1 \pm 4.5$ & 0.586 \\
\hline SF-36 & $83.1 \pm 6.3$ & $83.5 \pm 8.2$ & 0.921 \\
\hline ROM (degree) & & & \\
\hline $\begin{array}{l}\text { 6-month } \\
\text { extension }\end{array}$ & 0 & $0.3 \pm 1.3$ & 0.083 \\
\hline Flexion & $134.1 \pm 7.9$ & $132.4 \pm 8.3$ & 0.411 \\
\hline Final extension & 0 & 0 & \\
\hline Flexion & $138.5 \pm 4.4$ & $136.0 \pm 4.8$ & 0.249 \\
\hline
\end{tabular}

Abbreviations: HKA, hip-knee-ankle; KA, kinematically aligned; KS, Knee Society; MA, mechanically aligned; MCS, mental component summary; PCS, physical component summary; ROM, range of motion; SF, Short Form; WOMAC, Western Ontario and McMaster Universities.

Note: Values are presented as mean \pm standard deviation. The statistical significance was set at $p<0.05$.

KA TKA. ${ }^{6,7}$ These results could be explained by the native orientation of the distal femur and the proximal tibia. Normal value of mechanical lateral distal femoral angle and medial proximal tibial angle were known to be 88 and 87 degrees, respectively. ${ }^{16}$ Therefore, a femoral component valgus of 2.5 degrees and tibial component varus of 2.5 degrees in our KATKA could closely reproduce native orientation of the distal femur and proximal tibia. In this study, femoral valgus and tibial varus orientation in KA TKA resulted in more parallel joint line to the floor compared with MA TKA. Similar results were observed in studies by Ji et $\mathrm{al}^{17}$ and Matsumoto et al ${ }^{18}$ that compared postoperative JLOA between KA and MA TKA. Considering that JLOA of healthy patient is $0.3 \pm 2.0$ degrees in the study by Victor et $\mathrm{al}^{19}$ and $0.2 \pm 1.1$ degrees in the study by Ji et al, ${ }^{17}$ KA TKA seems to reproduce joint-line orientation more closely to prearthritic status compared with MA TKA.

Another important finding in this study was that KA TKA showed more internally rotated femoral and tibial components with similar rotational mismatch compared with MA TKA. Nedopil et $\mathrm{a}^{20}$ have also reported that incidence of rotational mismatch between femoral and tibial components in KATKA is less frequent than in MATKA. This finding could be related with the results of the intraoperative kinematic study by Maderbacher et $\mathrm{al}^{21}$ that reported more natural rotational movement of KA TKA compared with MA TKA. Besides rotational alignments of the tibial and femoral components, various factors could affect rotational movement of tibia after TKA. Therefore, further studies were necessary to clarify the relationships between rotational alignments of components and rotational movement of tibia. 
In the evaluation of patellofemoral kinematics, patella tilt angle was increased in KA TKA compared with MA TKA. However, patellar tilting angle in KA TKA was closer to preoperative status rather than that of MA TKA. Although more internal rotation of femoral component in KA TKA could increase the risk of patellofemoral maltracking theoretically, recent studies on patellofemoral kinematics between KA and MA TKAs have reported the results against this assumption. Keshmiri et $\mathrm{al}^{22}$ reported more physiologic patellar kinematics in KA TKA compared with MA TKA similar with our results in patellar tilting pattern. In a study by Koh et al, ${ }^{23}$ KA TKA restored patellar kinematics closer to normal knee compared with MA TKA. Different tibiofemoral kinematics or more laterally positioned distal trochlear groove in KA TKA could be the reason of this phenomenon, and further study is necessary to clarify this assumption.

The anthropometric measurement of distal femoral cutting surface might be changed according to characteristics of KA and MA. These results may be due to the relative internal rotation of femoral components in KATKA and use of the posterior referencing system. MA TKA showed a clear grand piano sign, whereas KA TKA was closer to the butterfly sign. There was no statistically significant difference in zones $C, D$, and $E$; however, the length of distal femoral cutting surface tended to be longer than MA TKA in KA TKA. These results were due to the valgus cutting of the distal femoral condyle in KA TKA. In terms of mean difference between implant and distal femoral cutting surface, zone A showed KA TKA that was less overhang than MA TKA and zone B showed KA TKA that was more overhang than MA TKA. These results are also due to the relative internal rotation of femoral components in KA TKA. There was no statistically significant difference in zones $\mathrm{C}, \mathrm{D}$, and $\mathrm{E}$; however, the mean difference between implant and distal femoral cutting surface tended to more underhang than MA TKA in KA TKA. These results were also due to the valgus cutting of the distal femoral condyle in KA TKA.

\section{Limitations}

This study has some limitations. First, in this study, KA and MA TKAs were performed using posterior and anterior referencing system, and referencing system can affect anterior femoral cut configuration. However, there was no definite notching or overstuffing case in MA TKA. Therefore, we believe that the effect of different referencing system may be minimal in this study. Second, all MA TKAs were performed with posterior stabilizing design. Therefore, the result of this study can be different from the comparison between KA and MA TKAs with cruciate retaining prostheses. However, considering that bone cutting was performed with identical surgical technique without influence of posterior cruciate ligament status in all groups, comparison of coronal and rotational alignment can be interpreted as difference between KA and MA TKAs. Third, our analysis was restricted to the alignment and morphologic analysis and other aspects, such as biomechanics and longevity, were not considered. Our follow-up period is short to discuss long-term survival and longevity.

\section{Conclusion}

KATKA showed more parallel JLOA to floor, closer patellar tilt to preoperative status, and better anterior flange fitting that can reproduce more natural knee kinematics compared with MA TKA. Although clinical outcomes assessed by conventional evaluating tools were similar between two groups, further evaluation focusing on the patellofemoral symptoms or unawareness of TKA is necessary to clarify the clinical benefit of KA TKA.

\section{Funding}

None.

Conflict of Interest

None declared.

\section{References}

1 Poilvache PL, Insall JN, Scuderi GR, Font-Rodriguez DE. Rotational landmarks and sizing of the distal femur in total knee arthroplasty. Clin Orthop Relat Res 1996;(331):35-46

2 Miller MC, Berger RA, Petrella AJ, Karmas A, Rubash HE. Optimizing femoral component rotation in total knee arthroplasty. Clin Orthop Relat Res 2001;(392):38-45

3 Kim JI, Jang J, Lee KW, Han HS, Lee S, Lee MC. Anterior tibial curved cortex is a reliable landmark for tibial rotational alignment in total knee arthroplasty. BMC Musculoskelet Disord 2017;18(01):252

4 Howell SM, Papadopoulos S, Kuznik K, Ghaly LR, Hull ML. Does varus alignment adversely affect implant survival and function six years after kinematically aligned total knee arthroplasty? Int Orthop 2015;39(11):2117-2124

5 Howell SM, Howell SJ, Kuznik KT, Cohen J, Hull ML. Does a kinematically aligned total knee arthroplasty restore function without failure regardless of alignment category? Clin Orthop Relat Res 2013;471(03):1000-1007

6 Yoon JR, Han SB, Jee MK, Shin YS. Comparison of kinematic and mechanical alignment techniques in primary total knee arthroplasty: a meta-analysis. Medicine (Baltimore) 2017;96(39):e8157

7 Lee YS, Howell SM, Won YY, et al. Kinematic alignment is a possible alternative to mechanical alignment in total knee arthroplasty. Knee Surg Sports Traumatol Arthrosc 2017;25(11): 3467-3479

8 Guy SP, Farndon MA, Sidhom S, Al-Lami M, Bennett C, London NJ. Gender differences in distal femoral morphology and the role of gender specific implants in total knee replacement: a prospective clinical study. Knee 2012;19(01):28-31

9 Kim YH, Choi Y, Kim JS. Comparison of standard and genderspecific posterior-cruciate-retaining high-flexion total knee replacements: a prospective, randomised study. J Bone Joint Surg Br 2010;92(05):639-645

10 Hutt J, Massé V, Lavigne M, Vendittoli PA. Functional joint line obliquity after kinematic total knee arthroplasty. Int Orthop 2016;40(01):29-34

$11 \mathrm{Kim} \mathrm{JH}$, Yoo BW, Kim CW. Influence of the rotational alignment of the femoral and patellar components on patellar tilt in total knee arthroplasty. Knee Surg Relat Res 2015;27(03):163-167

12 Castelli CC, Falvo DA, Iapicca ML, Gotti V. Rotational alignment of the femoral component in total knee arthroplasty. Ann Transl Med 2016;4(01):4

13 Saffi M, Spangehl MJ, Clarke HD, Young SW. Measuring tibial component rotation following total knee arthroplasty: what is the best method? J Arthroplasty 2019;34(7S):S355-S360

14 Fong TS, Kim SC, Kim JE, Lee ES, Kim TW, Lee YS. Morphometric assessment of resected femoral cut surface in korean knees and its 
fitting with western-designed femoral components. J Arthroplasty 2019;34(09):1929-1937

15 Howell SM, Papadopoulos S, Kuznik KT, et al. Accurate alignment and high function after kinematically aligned TKA performed with generic instruments. Knee Surg Sports Traumatol Arthrosc 2013;21(10):2271-2280

16 Paley D, Pfeil J. [Principles of deformity correction around the knee]. Orthopade 2000;29(01):18-38

17 Ji HM, Han J, Jin DS, Seo H, Won YY. Kinematically aligned TKA can align knee joint line to horizontal. Knee Surg Sports Traumatol Arthrosc 2016;24(08):2436-2441

18 Matsumoto T, Takayama K, Ishida K, et al. Radiological and clinical comparison of kinematically versus mechanically aligned total knee arthroplasty. Bone Joint J 2017;99-B(05):640-646

19 Victor JM, Bassens D, Bellemans J, Gürsu S, Dhollander AA, Verdonk PC. Constitutional varus does not affect joint line orientation in the coronal plane. Clin Orthop Relat Res 2014;472(01): 98-104
20 Nedopil AJ, Singh AK, Howell SM, Hull ML. Does calipered kinematically aligned tka restore native left to right symmetry of the lower limb and improve function? J Arthroplasty 2018;33(02): 398-406

21 Maderbacher G, Keshmiri A, Krieg B, Greimel F, Grifka J, Baier C. Kinematic component alignment in total knee arthroplasty leads to better restoration of natural tibiofemoral kinematics compared to mechanic alignment. Knee Surg Sports Traumatol Arthrosc 2019;27(05):1427-1433

22 Keshmiri A, Maderbacher G, Baier C, Benditz A, Grifka J, Greimel F. Kinematic alignment in total knee arthroplasty leads to a better restoration of patellar kinematics compared to mechanic alignment. Knee Surg Sports Traumatol Arthrosc 2019;27(05): 1529-1534

23 Koh IJ, Park IJ, Lin CC, et al. Kinematically aligned total knee arthroplasty reproduces native patellofemoral biomechanics during deep knee flexion. Knee Surg Sports Traumatol Arthrosc 2019; 27(05):1520-1528 